Title: The effect of proximity of COVID-19 test facilities on test uptake: Two quasiexperimental trials

Article Type: Randomised Controlled Trial

Authors: Sanders, J.G. ${ }^{1,2^{\wedge *}}$, Zomer. C. ${ }^{1,3^{*}}$, Hoekstra, R.H.A. ${ }^{4}$, de Ron. J. ${ }^{4}$, Blanken, T.F. ${ }^{4}$, Epskamp, S. ${ }^{4}$, van Dijken, K. S. ${ }^{1,5}$, Gerkema, M.H. ${ }^{6}$, Hart, L. ${ }^{6}$, Visser, O. ${ }^{3,6}$, Borsboom, D. ${ }^{4}$ de Bruin, M $^{1,3}$.

${ }^{1}$ Corona Behavioural Unit, National Institute of Public Health and the Environment, Bilthoven, The Netherlands

${ }^{2}$ Department of Psychological and Behavioural Science, London School of Economics and Political Sciences

${ }^{3}$ Radboud University Medical Center, Institute of Health Sciences, Nijmegen, The Netherlands

${ }^{4}$ Department of Psychological Methods, University of Amsterdam, Amsterdam, The Netherlands

${ }^{5}$ Municipal Health Service Flevoland region, The Netherlands (GGD Regio Flevoland)

${ }^{6}$ Municipal Health Service Utrecht region, The Netherlands (GGD Regio Utrecht)

*Joint first authorship

'Corresponding author: jet.sanders@rivm.nl

\title{
Summary
}

Background Test, Trace and Isolate (TTI) is a key strategy in the SARS-CoV-2 pandemic response. There is limited experimental evidence on how to improve uptake of COVID-19 testing. In this study we manipulate test site proximity to evaluate its impact on test uptake.

Methods We conducted two quasi-experimental studies (from February 8th to March 21st, 2021) during a community-wide testing initiative in The Netherlands. In Study 1 we placed a test site in one village (reducing distance to test site from $8.4 \mathrm{~km}$ to $800 \mathrm{~m}$ ) but not in a matched control village (distance to test site $9.9 \mathrm{~km}$ ). In Study 2 a mobile test bus alternated between two areas codes, changing distance to the test site from $3.5 \mathrm{~km}$ to $200 \mathrm{~m}$ and from $1.6 \mathrm{~km}$ to $850 \mathrm{~m}$. The trial is registered at the Netherlands Trial Register (number NL9365).

Findings In Study 1 ( $\mathrm{n}=11,317$ eligible inhabitants), a logistic regression controlling for baseline differences ( $8.6 \%$ control vs $10 \%$ intervention) found a significant effect of reducing test site proximity (17.5\% control vs $29 \%$ intervention) [Odds Ratio $=1.84\{95 \%$ CI 1.68-2.02\}]; $p<0.0001]$. In Study 2 ( $n=1,880$ eligible inhabitations), a Poisson regression revealed higher test uptake when the mobile test bus was present (33.6\% when absent vs $42.6 \%$ when present, Incidence Rate Ratio $=1.35$, [95\% CI 1.14 $1.59] ; p<0.0001)$.

Interpretation Reducing the distance to COVID-19 test facilities increased test uptake, at least when the distance was reduced from an average of $3.5 \mathrm{~km}$ to 200 metres. Localising test facilities can substantially increase testing for COVID-19, and thus the effectiveness of TTI in general.

Funding The Ministry of Health, Welfare and Sport (VWS) of the Netherlands. 
Keywords: proximity, test uptake, COVID-19, SARS-CoV-2, distance, mobile services, accessibility

\section{Research in context}

Evidence before this study We reviewed systematic reviews prior to, and individual publications and reports since the start of the COVID-19 pandemic (March 2020) for proximity interventions with a primary outcome of test uptake. We searched Google Scholar and PubMed for "test uptake" or "screening" in addition to "distance" or "proximity" or "access" and "systematic review" or "SARS-CoV2" or "COVID-19". On review of abstracts, we selected studies published in English and identified studies focused on the influence of distance to testing sites on test uptake (any condition). The identified evidence was observational, conducted in rural areas in low-resource settings or amongst disadvantaged populations, and focused on screening for conditions such as HIV or cancer. Their evidence does suggest distance to test sites is a barrier to test or screening uptake. There was one study that provided experimental evidence for low-resource countries: the randomised placement of HIV testing centres at distances greater than $1.5 \mathrm{~km}$ reduced attendance to HIV screenings by $6 \%$ in Malawi. Experimental evidence was not identified for high-resource countries. With regards to COVID-19 test facility proximity, two policy reports of observational studies note that the number of testing facilities in a region was correlated with test uptake, and one recent publication shows a negative association between test uptake and distance to testing site. Limitations of this evidence-base are that proximity and test uptake could well be confounded by demographic markers, such as deprivation level. Evidence from lowresource settings and other conditions might not generalise to COVID-19 testing in high-resource settings.

Added value of this study Our research provides quasi-experimental evidence from two pragmatic trials showing that closer proximity of test facilities substantially increases COVID-19 test uptake, with benefits observed when the distance to test locations was reduced from an average of 8.5 kilometres to 800 meters, and from 3.5 kilometres to 200 meters; but not with a smaller reduction (from 1.6 kilometres to 800 metres).

Implications of all available evidence To enhance or maintain good uptake of COVID-19 testing, it is important to facilitate behaviour by maintaining a fine-mazed network of test facilities - preferably substantially closer than 3.5 kilometres of people's homes. This evidence also contributes to the wider testing and screening literature in high-resource settings, which lacks experimental evidence. 


\section{Introduction}

The SARS-Cov2 pandemic has had a profound impact on human life worldwide, with (at the time of submission) over 412 million registered cases, and over 5.8 million registered deaths. ${ }^{1}$ Test, Trace, and Isolate (TTI) is a key strategy in the COVID-19 pandemic response. ${ }^{2,3}$ Test uptake is essential for monitoring the progress of the pandemic and an effective TTI strategy. National surveys demonstrate that only $20-60 \%$ of the eligible population (individuals with symptoms indicative of COVID-19) get tested. ${ }^{4,5}$ One reason people self-report for not getting tested is a lack of accessibility to (free) testing sites. ${ }^{6-9}$

Prior to the COVID-19 pandemic, studies have considered proximity of testing sites in various health contexts, such as for HIV, STI, cognitive impairment, TB and cancer screenings. Mostly, studies rely on surveying self-reported barriers or use geographical mapping to examine test-uptake by looking at the distance between test site and people's home address. ${ }^{10-17}$ These observational studies have consistently identified negative associations for distance to site or lack of transport, and test uptake. ${ }^{11}$ For example, a study in rural China found that test uptake for HIV decreased by $10 \%$ for every average kilometre of distance. ${ }^{12}$ Another study found a decrease in breast cancer screening with increased distance to clinic in North Derbyshire, UK. ${ }^{13}$ In fact, one systematic review concludes that geographic and transportationrelated access is an important barrier not only for testing, but across the continuum of care ${ }^{14}$ However, distance to site may well be confounded with socioeconomic markers ${ }^{15,16}$, and experimental evidence is scarce. We identified only one experimental study: the randomised placement of HIV testing centres at distances greater than $1.5 \mathrm{~km}$ reduced attendance to HIV screenings by $6 \%$ in Malawi. ${ }^{17}$ Understandably, most studies focus on rural areas in low-resource countries.

Since the start of the pandemic, in two community-wide testing initiatives (in Tirol, Austria ${ }^{18}$ and in Liverpool, United Kingdom ${ }^{19}$ ) researchers evaluated the relationship between distance to COVID-19 test sites and test uptake. In community wide testing, people are invited to repeatedly test for COVID-19 regardless of symptoms, to examine whether this strategy helps to suppress onward transmission. Both projects show a positive association between number of testing centres per capita and number of tests per region, and a negative association between distance to testing site and tests per area code. In both studies reduced access to testing sites confounded with socioeconomic markers or area deprivation scores. Other noted confounds include differences in education level and trust in government between compared populations. Hence, experimental evidence on the effects of changing distance to COVID-19 testing sites on test uptake is currently lacking - but this also applies to experimental evidence on test and screening uptake in high-resource settings for other conditions.

In two pragmatic trials in The Netherlands, we were able alter the proximity of the testing site and examined changes in COVID-19 test uptake in the context of community-wide testing programmes. In these studies we test the hypothesis that closer proximity of testing sites -in a high-resource country with excellent infrastructure- affects the uptake of COVID-19 testing. 


\section{Methods}

Study design, and participants

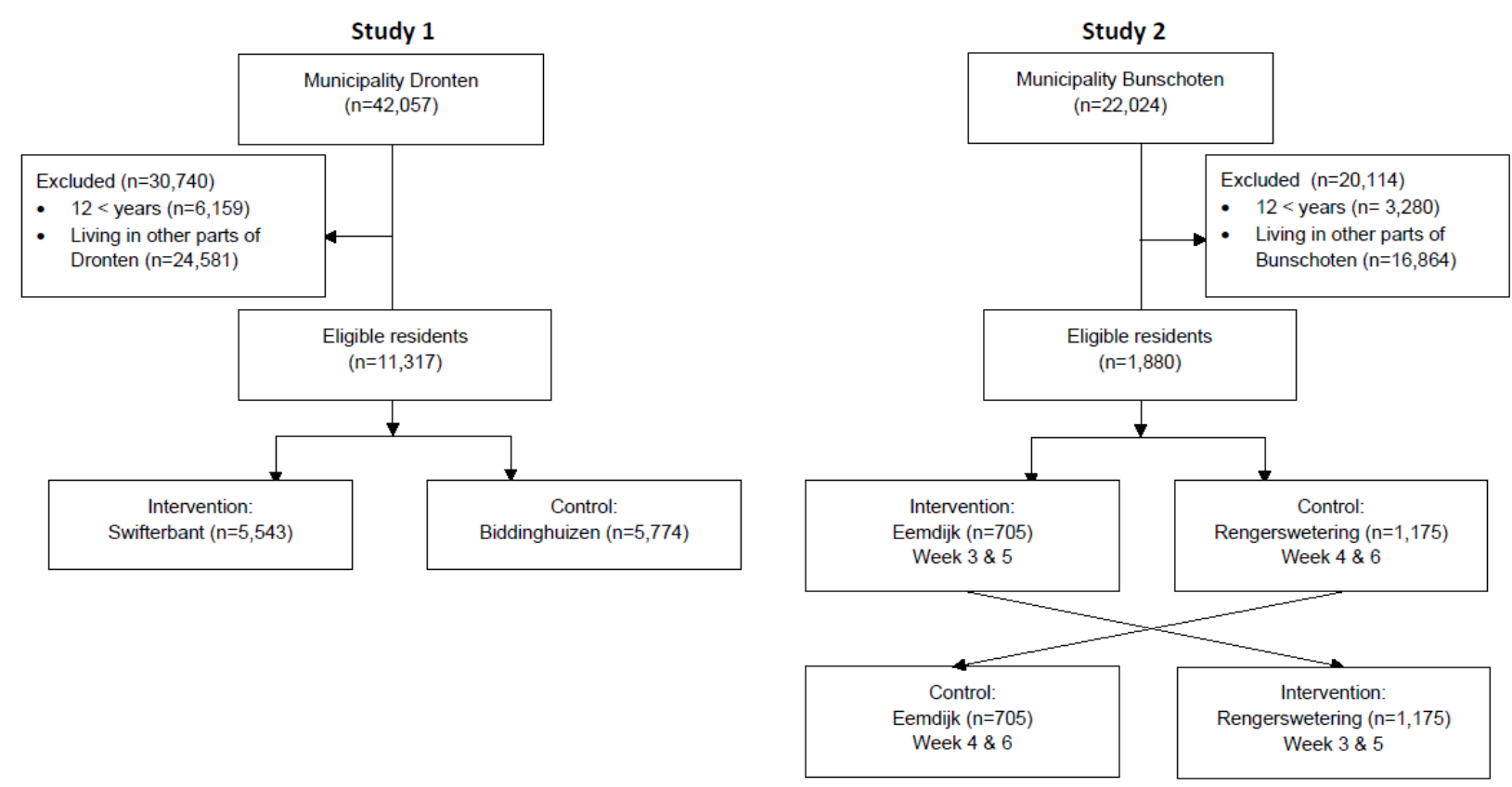

Figure 1. Trial profiles in the municipality of Dronten (Study 1) and municipality of Bunschoten (Study 2).

In two pragmatic, quasi-experimental trials we varied the proximity of testing sites in the context of two community-wide testing projects in the Netherlands. Study 1 took place in the municipality of Dronten (7-day average infection rate during trial 16.2/100,000 positive tests) and Study 2 in the municipality of Bunschoten (7-day average infection rate during trial: $21.6 / 100,000$ positive tests). ${ }^{20}$

In the municipality of Dronten (Study 1), we identified two villages (Swifterbant and Biddinghuizen) with a comparable number of inhabitants $(5,543$ versus $5,774,<12$ years) on a number of demographic markers such as age, gender, and marital status (See Supplementary Table 1), and distance to test sites (average 8.4 [range 7.9-9.4] km versus 9.9 [range 9.3-10.5] km, respectively). We placed an additional test site in Swifterbant (experimental arm) and made no change for the comparator Biddinghuizen.

In Study 2, in the municipality of Bunschoten, residents of the area codes Eemdijk and Rengerswetering were allocated to a crossover design, where we alternated the presence of a mobile test bus during a period of 4 weeks in both neighbourhoods (weekly rotation). Residents aged 6-12 years old were also invited to test frequently for COVID-19 as part of a pilot on a new child-approved testing method. Their data were excluded from the current proximity study.

We used data collected routinely by municipal health services. Citizens were informed by their municipalities about the community-wide testing initiative and that research would be conducted to examine test uptake. No person identifiers such as demographics were collected from study participants so that it was not necessary to obtain informed consent from individual residents whose test data were 
included in the trial. Ethical approval was obtained from the Department of Psychology, University of Amsterdam [n2021-PML-13061], and The Medical Review Ethics Committee of the National Institute of Public Health and the Environment (RIVM) of the Netherlands provided a declaration indicating the trial was exempt from medical ethical review [G\&M-514]. The study was pre-registered at the Netherlands Trial Register [NL9365], and a data analysis plan was registered on the Open Science Framework (10.17605/OSF.IO/4CN85).

\section{Intervention allocation}

In Study 1, an additional testing site was placed in the centre of Swifterbant $(n=5,543)$, reducing the average distance to testing site from $8.4 \mathrm{~km}$ (range $7.9 \mathrm{~km}-9.4 \mathrm{~km}$ ) to $800 \mathrm{~m}$ (range $60 \mathrm{~m}-1.9 \mathrm{~km}$ ) for its residents (Intervention). Residents were informed about the existence of this new test site through invitation letter and local news media. As comparator we used the village Biddinghuizen $(n=5,774)$, were no modifications were made in distance to test site (average distance $9.9 \mathrm{~km}$; range $9.3 \mathrm{~km}-10.5 \mathrm{~km}$ ).

In Study 2, a mobile test bus alternated weekly for four weeks of the community-wide testing initiative between Eemdijk ( $n=705)$, an adjacent area without testing facilities, and Rengerswetering $(n=1,175)$, central to the town (thus close to the town's main testing site). Presence of the mobile test bus decreased the average distance to the test site from $3.5 \mathrm{~km}$ (range: $3.5 \mathrm{~km}-3.6 \mathrm{~km}$ ) to $200 \mathrm{~m}$ (range: $190 \mathrm{~m}-220 \mathrm{~m}$ ) for residents of Eemdijk and from $1.6 \mathrm{~km}$ (range: $1.2 \mathrm{~km}-2.2 \mathrm{~km}$ ) to $850 \mathrm{~m}$ (range: $200 \mathrm{~m}-1200 \mathrm{~m}$ ) for residents of Rengerswetering. Although initially we planned to conduct this study for the six week period of the community-wide testing project, heavy snowfall during the first two week interfered with intervention implementation (the test bus could not be moved) and were thus excluded from the analysis.

\section{Procedures}

Prior to the community wide testing, citizens of the Netherlands were asked to get PCR tested if they showed COVID-19 related symptoms or had been in close contact with a person with COVID-19. During the trial period, all residents of participating municipalities were invited to get tested repeatedly regardless of symptoms or close contact. Community wide testing projects ran for six weeks from February 8 to March 21st, 2021 in Study 1; and from February 8 to March 19th, 2021 in Study 2. Testing sites were open every day of the week in Study 1, and six days a week in Study 2 (Sunday closure for religious reasons). In Study 1, people were asked to get tested three times per week during the six week trial period. In the municipality of Bunschoten, people were asked to get tested as many times as they would like during the six-week period.

Residents in both municipalities were encouraged to partake in community wide testing via news media, social media, billboards, and endorsements by public figures in the weeks leading up to, and during the trials. In Study 1, the municipality sent invitation letters in three batches on the 9th, 11th, and 13th of February 2021. In Study 2, these were sent out in two batches on the 4th and 5th of February 2021. Each batch was sent to a random selection of postal codes to spread the number of anticipated calls for appointments. The invitation offered basic information, describing the importance of participating in community-wide testing and partaking in the trials. In Study 1 residents were informed of the nearest testing sites, in Study 2 addresses of all testing sites were communicated and when the mobile test bus would be where. 
Test appointments were made over the phone and could be booked for other family members if these were present to agree. In Study 1, residents could book only one appointment at a time. In Study 2, residents could book multiple appointments in advance. As a means to boost test uptake, from week 3 (Study 1) and week 4 (Study 2) onwards, residents could get tested without making an appointment first (to boost uptake). This adjustment was paired with a reminder letter. These were sent in batches on February 9th, 11th, and 13th for Study 1 and on February 4th and 5th for Study 2.

Residents who tested without symptoms were not required to quarantine in anticipation of their results. Once a resident had completed a PCR test, they received their test results via a secured online platform within 48 hours of the test. Residents were notified when their results were ready via email, alongside an access code for the secure platform. If a resident tested positive, local health authorities contacted to aid in tracing and isolating procedures.

\section{Outcomes}

Municipal health authorities routinely record person identifiers and COVID-19 test results. For Study 1, baseline test data were used to control for differences between villages prior to the trial. Baseline testing data were comprised of the number of unique residents who got tested in the prior 6 weeks. The primary outcome consisted of two parts: the number of individuals who tested for COVID-19 and the number of times a unique individual tested during the 6-week trial period (combined reflecting the total number of tests conducted). In Study 2 no baseline data was used as residents across the two area codes were exposed to both treatments. The primary outcome variable consisted of the total number of tests completed.

\section{Statistical analysis}

For both studies, for the period during the trial, publicly available records on postal codes, municipality area codes, and the number of individuals per household (public records) were matched with the anonymised number of people who tested per address, the number of tests completed per unique individual at that address, the date of their visit, and testing site at which the test was completed. As data only pertained to tested residents, we imputed zero's (not tested) for the residents who did not appear in the database, using the total number of residents per area code. The data used for the 6 weeks prior to the trial in Study 1 could not be matched to an individual level.

For Study 1, we first estimated the effect of proximity on the number of unique residents who were tested (DV) using a log-linear regression model comparing village Swifterbant (experimental) and

Biddinghuizen (comparator) (Treatment, IV1) prior to and during the trial period (Time, IV2), and we included an interaction effect between treatment and time (IV3) to test whether the change in testing from baseline to follow-up was larger in the experimental than comparator arm. To allow for a comparison with study 1, we assess the effect of proximity on the number of tests per person, by comparing the average number of completed tests per person (DV) between villages (IV) during the trial period using a Mann-Whitney-Wilcoxon test. In post-hoc analyses we compared the individuals who completed testing (DV) once, twice, or thrice between the two villages (IV) using Fisher exact tests, controlled for multiple comparisons. 
In Study 2, we used a Poisson regression model of presence (experimental) or absence (comparator) of the mobile test bus (IV), controlling for district (CV), and effect of time (by week; CV) on total test frequency (DV). In a post-hoc analysis, we assessed treatment effects for each neighbourhood separately, using a McNemar's Chi-squared test of test uptake in each area code (DV) when the test bus was absent or present (IV).

\section{Role of the funding source}

The study was funded by the Ministry of Health, Welfare, and Sport of the Netherlands. The funder played no role in study design, data collection, data analysis, data interpretation, or writing of the report.

\section{Results}

In Study 1, 10\% $(n=552)$ of residents in the experimental arm versus $8.6 \%(n=496)$ in the comparator were tested during the six weeks prior to the trial, before community-wide testing was initiated. During the trial period $29 \%(n=1,606)$ of Swifterbant residents were tested (experimental), relative to $17.5 \%$ $(\mathrm{n}=1,010)$ of Biddinghuizen residents (control; see Figure 2A). A log-linear regression (see Table 1 for detail) demonstrates a main effect of time $(b=0.71, Z=12.97, p<0.0001 ; R R=2.04,95 \% \mathrm{CI}[1.83-2.27])$, no main effect of condition $(b=0.11, \mathrm{Z}=1.73, \mathrm{p}=0.08 ; \mathrm{RR}=1.11,95 \% \mathrm{CI}[0.99-1.26])$ and an interaction effect between time and condition $(b=0.36, Z=4.84, p<0.0001 ; R R=1.43,95 \% \mathrm{CI}[1.24-1.65])$. Hence, the introduction of community-wide testing resulted in an increase in testing in both arm (the main effect time), and this increase was substantially larger in the experimental arm (the interaction term). The overall number of residents in the municipality of Dronten who were tested increased from $7.7 \%$ $(n=3,250)$ in the six-week prior to community-wide testing to $27.8 \%(n=10,146)$ during the six-week trial period.

Comparing the effects of proximity on the number of tests per person, we found that residents of Swifterbant (experimental arm, $\mathrm{M}=0.55, \mathrm{SD}=0.99$ ) tested more often than in Biddinghuizen (comparator; $\mathrm{M}=0.27, \mathrm{SD}=0.68 ; \mathrm{W}=17994330, p<0.0001 ; r=-0.15,95 \% \mathrm{CI}[-0.16--0.13]$ ). The effects of proximity increased with the frequency of testing, from testing once (Swifterbant: 13.2\%, Biddinghuizen; 10.8\%; OR $=1.25,95 \%$ CI [1.12 -1.41], $p<0.001$ ) to testing twice (Swifterbant: $6.6 \%$, Biddinghuizen: $3.6 \%$; OR $=1.90,95 \%$ CI [1.59 -2.26], $p<0.0001$ ) to testing thrice (Swifterbant: $8.4 \%$, Biddinghuizen: 3.0\%; OR $=3.01,95 \%$ CI [2.52-3.62], $p<0.0001$; see Figure 2B). Overall, $14.0 \%$ of the residents in the municipality of Dronten tested once, $6.4 \%$ tested twice and $7.3 \%$ three times. 


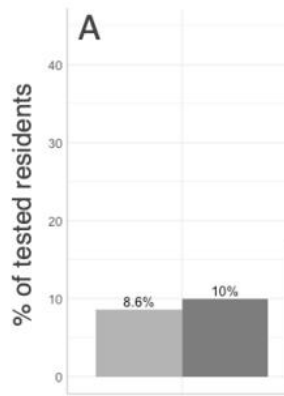

Prior to trial

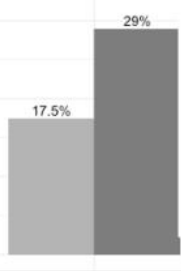

During trial

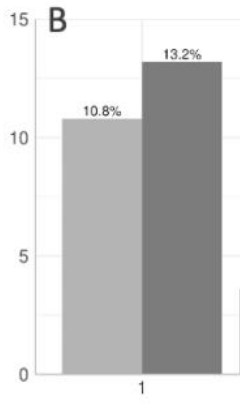

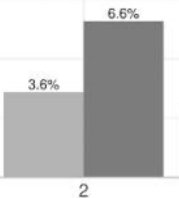

Number of times tested during trial

Table 1: Percentage of tested residents in the municipality of Dronten (Study 1) for Swifterbant (intervention) and Biddinghuizen (control) in $(\mathbf{A})$ the six weeks prior to and during the trial and $(\mathbf{B})$ during the trial period by the number of completed tests per person.

\begin{tabular}{|c|c|c|c|c|c|}
\hline Predictor & b (SD) & $\mathbf{Z}$ & $\mathbf{p}$ & $\begin{array}{l}\text { OR / } \\
\mathbf{R R}\end{array}$ & $95 \% \mathrm{CI}$ \\
\hline \multicolumn{6}{|l|}{ Location } \\
\hline Biddinghuizen (comparator) & \multicolumn{5}{|c|}{ reference } \\
\hline Swifterbant (experimental arm) & $0.11(0.06)$ & 1.73 & 0.08 & 1.11 & $0.99-1.26$ \\
\hline \multicolumn{6}{|l|}{ Time } \\
\hline Prior to trial (control) & \multicolumn{5}{|c|}{ reference } \\
\hline During trial (treatment) & $0.71(0.05)$ & 12.97 & $<0.0001$ & 2.04 & $1.83-2.27$ \\
\hline \multicolumn{6}{|l|}{ Interaction } \\
\hline Time $\mathrm{x}$ Location & $0.36(0.07)$ & 4.84 & $<0.0001$ & 1.43 & $1.24-1.65$ \\
\hline \multicolumn{6}{|l|}{ Pairwise comparisons } \\
\hline Prior: Swifterbant vs. Biddinghuizen & & & $<0.0001$ & 1.18 & $1.04-1.34$ \\
\hline During: Swifterbant vs. Biddinghuizen & & & $<0.0001$ & 1.84 & $1.68-2.02$ \\
\hline Swifterbant: prior vs. during & & & $<0.0001$ & 3.20 & $2.88-3.56$ \\
\hline Biddinghuizen: prior vs. during & & & $<0.0001$ & 2.26 & $2.01-2.53$ \\
\hline
\end{tabular}

Table 1. Logistic regression on test uptake in the municipality of Dronten (Study 1).

In Study 2 (municipality of Bunschoten), 375 tests were completed when the mobile test bus was present and 317 were completed when the mobile test bus was absent over a period of four weeks. This consisted of $18.5 \%$ of unique residents when the mobile test bus was present $(n=329)$ and $15.2 \%$ of unique residents when the mobile test bus was absent $(\mathrm{n}=259)$. Using a Poisson regression demonstrate a significant increase in the test uptake when the mobile testing facility was present $(\operatorname{LR}(1)=1,957, p=0.005$; OR $=$ 1.35, 95\% CI[1.15-1.59]; see Figure 3A and Table 2 for details). Pairwise comparisons using a McNemar's Chi-squared test show that the effect was driven by one neighbourhood (Eemdijk), where proximity was reduced from $3.5 \mathrm{~km}$ to $200 \mathrm{~m}$ [Present $(n=178): 25.2 \%$; Absent $(n=62), 8.8 \%$; OR $=3.46$, 
95\% CI[2 .03-5.89], $\left.X^{2}(1, n=705)=51.87, p<0.05\right]$, and not Rengerswetering, where proximity was reduced from $1.6 \mathrm{~km}$ to $850 \mathrm{~m}$ [Present $(\mathrm{n}=151): 12.9 \%$; Absent $(\mathrm{n}=197): 16.8 \%$; OR $=0.65,95 \%$ CI[ $\left.0.56-0.87, X^{2}(1, n=1,175)=9.95 p<0.05\right]^{2}$. See Figure 3B for details and Supplementary Table 3 for a separation by week). Analyses on test frequency were not possible due to low numbers.

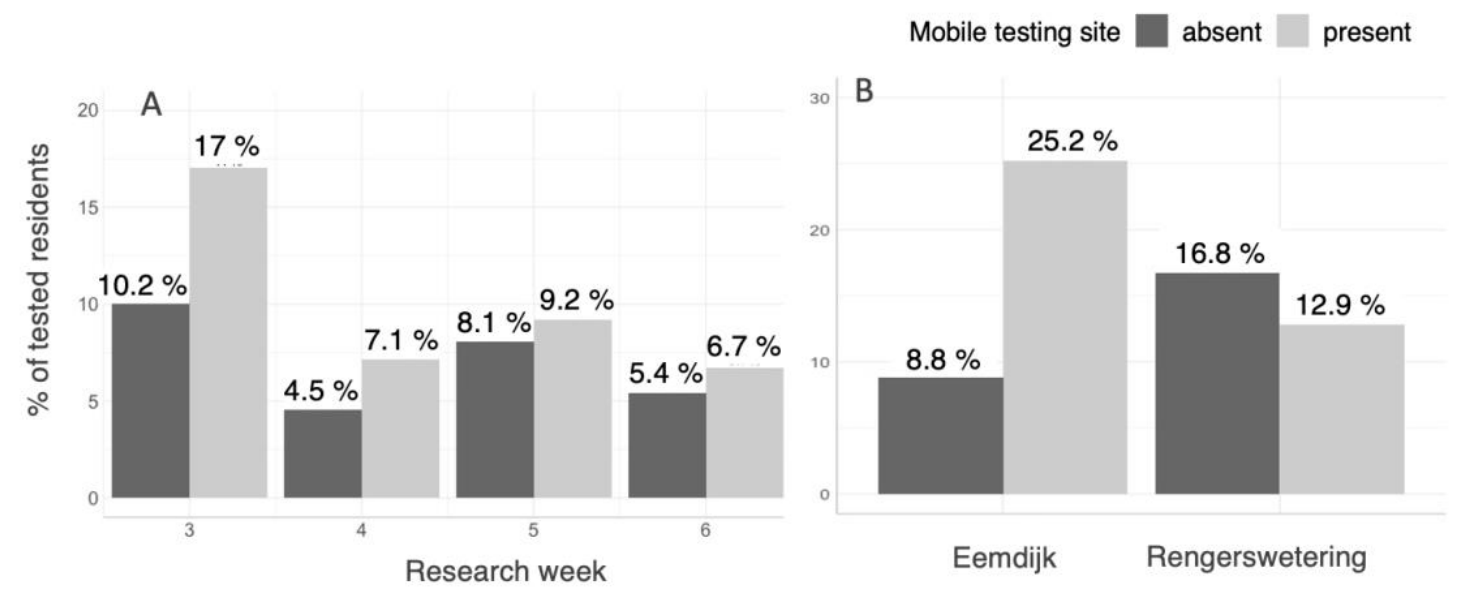

Figure 3: Test uptake in the municipality of Bunschoten during the last four weeks of the community wide testing initiative when a mobile testing facility rotated weekly between being present (intervention) and absent (control). (A) Percentage of tested residents per week and (B) by area code (Eemdijk and Rengerswetering).

\begin{tabular}{|c|c|c|c|c|c|}
\hline Predictor & b (sd) & $\mathbf{Z}$ & $\mathbf{p}$ & OR & $95 \% \mathrm{CI}$ \\
\hline \multicolumn{6}{|l|}{ Time in weeks } \\
\hline Week 3 & \multicolumn{5}{|c|}{ Reference } \\
\hline Week 4 & -0.81 & -7.13 & $<0.05$ & 0.45 & $0.38-0.52$ \\
\hline Week 5 & -0.37 & -3.74 & $<0.05$ & 0.69 & $0.58-0.82$ \\
\hline Week 6 & -0.73 & -6.6 & $<0.05$ & 0.48 & $0.41-0.57$ \\
\hline \multicolumn{6}{|l|}{ Mobile testing facility } \\
\hline Absent (control) & \multicolumn{5}{|c|}{ Reference } \\
\hline $\begin{array}{l}\text { Present } \\
\text { (intervention) }\end{array}$ & 0.3 & 3.59 & $<0.05$ & 1.35 & $1.15-1.59$ \\
\hline
\end{tabular}




\begin{tabular}{|l|l|l|l|l|l|}
\hline District \\
\hline Eemdijk & \multicolumn{5}{|c|}{ Reference } \\
\hline Rengerswetering & 0.23 & 2.79 & $<0.0001$ & 1.26 & $1.07-1.48$ \\
\hline
\end{tabular}

Table 2. Poisson regression on test uptake in the municipality of Bunschoten (Study 2).

\section{Discussion}

In two pragmatic, quasi-experimental trials conducted during community-wide testing programmes, we found that closer proximity to a test site resulted in higher test uptake: more unique residents tested and did so more frequently. In Study 1, the odds of testing repeatedly increased from 1.25 [1.12 -1.41] for testing once to 3.01 [2.52-3.62] for testing three times. The effects observed were visible when the distance to test site or bus was altered substantially (Study 1: 800 meters for the experimental arm and 9.9 for the comparator; Study 2, neighbourhood 1 from 3.5 kilometres to 200 metres), but not when the initial distance was smaller and the treatment contrast more modest (Study 2, neighbourhood 2: from 1.6 kilometres to 850 metres). This study thus demonstrates experimentally that reducing distance to test site from at least 3.5 kilometres to 200 metres (within a neighbourhood or village), can substantially impact testing for COVID-19 in settings with good infrastructure and transportation options.

Previous observational studies on testing and screening uptake - including for COVID-19 - have reported associations between the number of test sites or test site vicinity, and test uptake. ${ }^{3-19}$ Much of this evidence is from low-resource settings or underserved population in high-income settings. One experimental study on screening for HIV reported observing an effect for test site vicinity as close as 1.5 kilometres in a low-income context. This distance reflects approximately 15-20 minutes walking and 3.5 kilometres approximately 15 min cycling (a common mode of transport in The Netherlands) ${ }^{10-17}$; although this could be coincidence as the Dutch municipal health services ask people whether they have access to a car to come to the test site. Combined, the observational and current quasi-experimental evidence demonstrate that maintaining a fine-mazed network of test sites is important, including for rural communities and less densely populated areas to ensure equal access to healthcare resources.

The current study has several limitations. First, it could be that residents in the comparator village of Study 1 were aware of the additional test site opened in the experimental village. In theory, this may have resulted in reactance and a lower willingness to participate in the community-wide testing programme. ${ }^{22}$ Second, the sample size in Study 2 was too small to test the hypotheses of test site proximity on frequency of testing. Finally, the projects ran during a period with two weeks of heavy snowfall, the minister of health visiting the Study 1 municipality, and a prime minister press conference (see Supplementary Table 2 for a detailed list of events). Although these contextual dynamics were similar for both treatment arms, it was not impossible to model the exact effect of these events on our outcomes.

In conclusion, test site proximity matters for COVID-19 test uptake, also in settings with good infrastructure and different modes of transportation. We recommend aiming for placing easily accessible sites well within 3.5 kilometres of communities. This approach may not only benefit test uptake in general but may show an even increased benefit for areas of higher deprivation, where base rates are 
generally lower. ${ }^{13-16}$ The effects observed here may well transfer to other preventive health services, such as COVID-19 vaccination uptake. ${ }^{23}$

\section{Ethical statement}

Ethical approval was obtained from the department of psychology, UvA 2021-PML-13061 and The Medical Review Ethics Committee declared the trial was not required to go through medical ethical review (number G\&M-514).

\section{Acknowledgements}

We would like to show our gratitude to Ineke Brust, Paula Weiland, Jeroen Verburg and Jean Paul Gebben (municipality of Dronten) and Reinate Hopman and Melis van de Groep (municipality of Bunschoten) for hosting the community wide testing trials and the municipality Health service Utrecht (Juul Tönis) and Flevoland (Herman Fortuin, Aldrik Dijkstra and Charlie van der Weijden) for their collaboration. We would like to thank Jasmijn Haverhals, Bart van Sambeek and Maren Schaatsbergen for coordinating all parties involved and we would thank our colleagues with the National Institute for Public Health and the Environment (RIVM) Anne Buitenhuis, Koen van der Swaluw, Riny Janssen, Jose de Sousa, Floor Kroese and Mariken Leurs from the RIVM for providing expertise in developing manuscript, and all members of the RIVM-IOPS advisory board (Casper Albers, Sacha Epskamp, Joost van Ginkel, Fabian Dablander) for their advice on statistical models.

\section{Funding statement}

This study is funded by the Ministry of Health, Welfare and Sport of the Netherlands

\section{Conflict of interest}

We declare no conflicts of interest.

\section{Author contributions}

All authors fulfilled the ICMJE authorship criteria. All authors critically reviewed and modified the manuscript, and have approved the final version of the manuscript.

J.G. Sanders*: conceptualization, methodology, validation, investigation, writing (original draft \& review and editing), supervision, project administration; C.L. Zomer*: conceptualization, methodology, validation, investigation, writing (original draft \& review and editing); J. de Ron: formal analysis, visualisation, writing (review and editing); R.H.A. Hoekstra: formal analysis, visualisation, writing (review and editing); T. F. Blanken.: conceptualization, methodology, formal analysis; S. Epskamp.: formal analysis; K. S. van Dijken: data curation; M.H. Gerkema: data curation; L.L. Hart: data curation, project administration; O. Visser: data curation, project administration, funding acquisition; D. Borsboom: conceptualization, methodology, formal analysis, validation, supervision; M. de Bruin: conceptualization, methodology, supervision, funding acquisition, writing (review and editing).

* equal contribution - shared first authorship

\section{References}


${ }^{1}$ WHO: World Health Organisation. Health Emergency Dashboard. [Internet] Available at: https://covid19.who.int/ [Accessed 26th January 2022].

${ }^{2}$ CDC: Centers for Disease Control and Prevention. Testing overview. [Internet]. Available at: https://www.cdc.gov/coronavirus/2019-ncov/hcp/testing-overview.html [Accessed 8th August 2021].

${ }^{3}$ European Centre for Disease Prevention and Control. COVID-19 testing strategies and objectives. 15 September 2020. ECDC: Stockholm; 2020.

${ }^{4}$ Sanders JG, Kroese F, de Bruin M, Zomer C, Buitenhuis A, Albers L, et al. Redenen om niet te laten testen bij klachten. RIVM Corona Behavioural Unit, Bilthoven; 2021. Available at: https://www.rivm.nl/documenten/redenen-om-niet-te-laten-testen

${ }^{5}$ Smith LE, Potts HWW, Amlôt R, Fear NT, Michie S, Rubin GJ et al. Adherence to the test, trace, and isolate system in the UK: results from 37 nationally representative surveys BMJ 2021; 372 :n608 doi:10.1136/bmj.n608.

${ }^{6}$ de Bruin M., Elberse J, Verweij A, Zonneveld M, Gorter A. Thuisblijven, testen en quarantaine. RIVM Corona Behavioural Unit, Bilthoven; 2020. Available at: https://www.rivm.nl/documenten/onderzoekthuisblijven-testen-en-quarantaine

${ }^{7}$ McElfish PA, Purvis R, James LP, Willis DE, Andersen JA. Perceived Barriers to COVID-19 Testing. Int J Environ Res Public Health 2021 Feb 25;18(5):2278. doi: 10.3390/ijerph18052278. PMID: 33668958; PMCID: PMC7956381.

${ }^{8}$ Chung S, Marlow S, Tobias N, et al. Lessons from countries implementing find, test, trace, isolation and support policies in the rapid response of the COVID-19 pandemic: a systematic review BMJ Open 2021;11:e047832. doi: 10.1136/bmjopen-2020-047832.

${ }^{9}$ Xu Y, Egan M, Shrestha P, Kelly S, Mottershaw A, Harper H. Practicalities are the most significant impediments to people getting a COVID vaccine - and the easiest to address. The Behavioural Insights Team. [weblog]. 2021. Available at: https://www.bi.team/blogs/practicalities-are-the-most-significantimpediments-to-people-getting-a-covid-vaccine-and-the-easiest-to-address/

${ }^{10}$ Cope AB, Powers KA, Serre ML, Escamilla V, Emch ME, Leone PA, et al. Distance to testing sites and its association with timing of HIV diagnosis. AIDS care 2016 Nov 1;28(11):1423-7.

${ }^{11}$ Kadota JL, Nabwire S, Nalugwa T, White JS, Cattamanchi A, Katamba A, et al. Patient Perspectives and Willingness to Accept Incentives for Tuberculosis Diagnostic Evaluation in Uganda.Value in Health Regional Issues 2021; 25: 48-56.

${ }^{12}$ Chen W, Zhou F, Hall BJ, Tucker JD, Latkin C, et al. Is there a relationship between geographic distance and uptake of HIV testing services? A representative population-based study of Chinese adults in Guangzhou, China. PLOS ONE 2017; 12(7): e0180801. https://doi.org/10.1371/journal.pone.0180801 
${ }^{13}$ Maheswaran R, Pearson T, Jordan H, et al. Socioeconomic deprivation, travel distance, location of service, and uptake of breast cancer screening in North Derbyshire, UK. Journal of Epidemiology \& Community Health 2006; 60: 208-212.

${ }^{14}$ Lankowski AJ, Siedner MJ, Bangsberg DR, Tsai AC. Impact of geographic and transportation-related barriers on HIV outcomes in sub-Saharan Africa: a systematic review. AIDS Behav 2014 Jul;18(7):1199223. doi: 10.1007/s10461-014-0729-8. PMID: 24563115; PMCID: PMC4047127.

${ }^{15}$ Nguyen CA, Chernew ME, Ostrer I, Beaulieu N. Comparison of healthcare delivery systems in low-and high-income communities. Am J Accountable Care 2019; 7(4): 11-8.

${ }^{16}$ Kanter GP, Segal AG, Groeneveld PW. Income Disparities In Access To Critical Care Services: Study examines disparities in community intensive care unit beds by US communities' median household income. Health Affairs 2020; 39(8): 1362-1367.

${ }^{17}$ Thornton RL. The Demand for, and Impact of, Learning HIV Status. American Economic Review 2008; 98(5): 1829-63. DOI: 10.1257/aer.98.5.1829

${ }^{18}$ Stillman S, Mirco T. Communities and Testing for COVID-19. CESifo Working Paper No. 8816, Available at SSRN: https://ssrn.com/abstract=3767688 or http://dx.doi.org/10.2139/ssrn.3767688

${ }^{19}$ Green M, Garcia-Finana M, Barr B. Burnside G, Cheyne CP. Hughes D. et al.. Evaluating Social and Spatial Inequalities of Large Scale Rapid Lateral Flow SARS-CoV-2 Antigen Testing in COVID-19 Management: An Observational Study of Liverpool, UK (November 2020 to January 2021). Lancet Reg Health Eur. 2021; Jul;6:100107. doi: 10.1016/j.lanepe.2021.100107.

${ }^{20}$ Corona Dashboard The Netherlands [2021, August 16], Retrieved from: https://coronadashboard.rijksoverheid.nl/gemeente/GM0303/positief-geteste-mensen

${ }^{21}$ GGD regio Utrecht. Eindrapportage Pilot Grootschalig Risicogericht Testen Bunschoten. 2021 Available at: https://www.bunschoten.nl/ flysystem/media/eindrapportage-pilot-bunschoten.pdf

${ }^{22}$ McCambridge J, De Bruin M, Witton J. The effects of demand characteristics on research participant behaviours in non-laboratory settings: a systematic review. PloS one. 2012 Jun 19;7(6):e39116.

${ }^{23}$ Zhang X, Tulloch J, Knott S, Allison R, Parvulescu P, Buchan I, García-Fiñana M, Piroddi R, Green M, Barr B. Evaluating the Impact of Using Mobile Vaccination Units to Increase COVID-19 Vaccination Uptake: A Synthetic Control Analysis for Cheshire and Merseyside, UK. UK (January 27, 2022). 2022 Jan 27. 